\title{
The protective effect of PK11195 on D-galactose-induced amnestic mild cognitive impairment in rats
}

\author{
Chen Ye ${ }^{1,2 \#}$, Lanying Lin ${ }^{1,2 \#}$, Peiling Zhang ${ }^{1,2}$, Yi Chen ${ }^{1,2}$, Jinghao Huang ${ }^{1,2}$, Xianzhong Lin ${ }^{1,2}$ \\ ${ }^{1}$ Department of Anesthesiology, the First Affiliated Hospital of Fujian Medical University, Fuzhou, China; ${ }^{2}$ Institute of Anesthesiology, Fujian \\ Medical University, Fuzhou, China \\ Contributions: (I) Conception and design: C Ye; (II) Administrative support: X Lin, J Huang; (III) Provision of study materials or patients: P Zhang, \\ Y Chen; (IV) Collection and assembly of data: L Lin; (V) Data analysis and interpretation: C Ye; (VI) Manuscript writing: All authors; (VII) Final \\ approval of manuscript: All authors. \\ \#These authors contributed equally to this work. \\ Correspondence to: Jinghao Huang, MD; Xianzhong Lin, MD, PhD. Department of Anesthesiology, the First Affiliated Hospital of Fujian Medical \\ University, Fuzhou, China. Email: 250832306@qq.com; lxzyjs@fjmu.edu.cn.
}

\begin{abstract}
Background: This study aimed to investigate the preventive effect of translocator protein $18 \mathrm{kDa}$ (TSPO) ligand PK11195 on amnestic mild cognitive impairment (aMCI), as well as its influence on astrocytes, in order to identify effective ways to prevent aMCI.

Methods: Male SD rats were randomly divided into control group (n=10), aMCI group (n=10), PK11195 group (n=10), PK11195 + D-gal group (n=10). The preventive effect of PK11195 on aMCI in rats was evaluated. The cognitive function of rats in four different treatment groups was determined using the Morris water maze (MWM), as well as whole-brain pathology and immunofluorescence of rat brain tissue.

Results: The results of the MWM behavioral test showed that rats pre-treated with PK11195 had improved escape latency and a higher number of platform crossings compared with the aMCI model rats. PK11195 was also shown to prevent the D-galactose (D-gal)-induced senescence of pyramidal cells in the hippocampal CA1 region and to inhibit the apoptosis of astrocytes. At the same time, compared with the aMCI model rats, the TSPO in the brain tissue of rats pretreated with PK11195 had a lower distribution density.
\end{abstract}

Conclusions: Our results prove that PK11195 can effectively prevent D-gal-induced decline of learning and memory function as well as inhibit abnormal changes of related cells.

Keywords: TSPO; PK11195; amnestic mild cognitive impairment (aMCI); astrocytes

Submitted Aug 10, 2020. Accepted for publication Sep 14, 2020.

doi: $10.21037 /$ atm-20-6157

View this article at: http://dx.doi.org/10.21037/atm-20-6157

\section{Introduction}

Early cognitive decline is increasing in prevalence with the growth of the aging population in China. Premature changes in cognitive function differ from those associated with normal or accelerated aging; although multidimensional cognitive decline occurs in these individuals with age, under normal circumstances, the damage to cognitive dimensions is more balanced. Mild cognitive impairment (MCI) is defined as a state in which the level of cognition declines beyond that of normal aging, but dysfunction has not yet occurred (1). Amnestic mild cognitive impairment (aMCI) is associated with frequent memory loss and progression to Alzheimer's disease (AD) (2). Epidemiological studies have reported that approximately half of patients who experience a mild decline in learning and memory abilities go on to develop $\mathrm{AD}$ (3). In 2018, healthcare, long-term care, and hospice services for $A D$ patients aged $\geq 65$ years in the United States totaled approximately $\$ 277$ billion (4). Therefore, 
exploring ways to prevent, diagnose, and treat aMCI is of immense value to reducing the occurrence of $\mathrm{AD}$.

aMCI is a normal part of the brain's natural aging process. Simulated aging has been widely used in in vivo studies; in AD mechanism research and drug model screening, chronic exposure to D-galactose (D-gal) has often been used to induce neurotoxicity in rat models $(5,6)$. With natural aging, the synthetic density of translocator protein $18 \mathrm{kDa}$ (TSPO) in the brains of animals continues to increase (7). TSPO is a common mitochondrial protein of $18 \mathrm{kDa}$ in size. It was originally named peripheraltype benzodiazepine receptor $(\mathrm{PBR})$ and is widely distributed in many organs and tissues including the brain, kidney, and heart (8). TSPO is mainly located on the mitochondrial membrane of external steroid synthesis cells. Among its main functions is the transportation of cholesterol into the mitochondria, which is a prerequisite for mitochondrial steroid synthesis (9). In a number of neuropathological studies, TSPO was used as a biomarker of neuroinflammation and reactive glial hyperplasia; consequently, various TSPO ligands have been developed as neuroimaging agents, including 11C-PK11195 in a positron emission tomography (PET) study of Parkinson's disease dementia (10). In addition, previous work in mice has shown that TSPO ligands may be a potential therapeutic intervention to slow the progression or even reverse $\mathrm{AD}$ neuropathology (11). Among them, PK11195 and Ro5-4864 are commonly used in the research field of brain related cognitive function, and have been proved to have pleiotropic neuroprotective effect. The TSPO ligand isoquinoline carboxamide (PK11195) previously has been shown to decrease microglial activation and inflammation (12). PK11195 can specifically bind to TSPO, but Ro5-4864 needs the assistance of other mitochondrial proteins to complete the binding of TSPO protein. In animal models of neurodegenerative diseases (13) and neuroinflammatory psychosis (14), TSPO ligands have been shown to have significant in vivo efficacy. These studies emphasized that TSPO ligands can be used for neuroprotection, limiting neuroinflammation, promoting glial cell regeneration, and treating nervous system dysfunction.

At present, studies on the relationship between the occurrence of aMCI during aging and the up-regulation of TSPO expression, as well as the potential preventive or therapeutic role of TSPO classic ligands in aMCI, are lacking. Age-associated increases in the expression of TSPO in the central nervous system (CNS) may affect the astrocytes accompanying CNS development. As supporters of important neurogenic physiological functions, astrocytes perform four main functions: (I) supporting and isolating the original structure of the central nervous system, such as neural tubes, in the embryonic period (15); (II) adjusting the concentration of internal and external ions and transmitting the second messenger; (III) ingesting, inactivating, and supplying neurotransmitters (16); and (IV) regulating nutritional repair and antioxidant effects $(17,18)$. To ensure normal neurophysiological function, the maintenance of the biological integrity of astrocytes are of great significance. Previous studies have confirmed that the mitochondria is the core structure involved in the regulation of apoptosis. Under the stimulation of various damage factors, the mitochondria can undergo osmotic translocation (19), eventually leading to the transfer of apoptotic proteins from the mitochondrial bilayer membrane into the cytoplasm (20).

Our previous study showed that subcutaneous injection of D-gal $(1,000 \mathrm{mg} / \mathrm{kg} / \mathrm{day})$ in rats can result in mild nerve pathological changes, cholinergic system function impairment, mild oxidative damage, and abated oxidation resistance, as well as slight decreases in spatial learning and memory abilities, with little impact on motivation and foraging behavior. Therefore, in order to explore the mechanism of the TSPO-induced pathophysiology of astrocyte morphology, we chose to induce brain aging in rats using the classic method of continuous injection of D-gal via the back of the neck; at the same time, the rats were administered repeated injections of TSPO ligand PK11195 via the intraperitoneal cavity to observe its effect on D-gal-induced cognitive function in aMCI.

We present the following article in accordance with the ARRIVE reporting checklist (available at http://dx.doi. org/10.21037/atm-20-6157).

\section{Methods}

\section{Ethical statement}

Experiments were performed under a project license (No.: 2018-321) granted by Fujian experimental animal association, in compliance with EU Directive 2010/63/EU for the care and use of animals.

\section{Animals}

Forty male Sprague-Dawley rats (age, 10-12 weeks; weight, $280 \pm 50 \mathrm{~g}$ ) were purchased from Shanghai Slake 


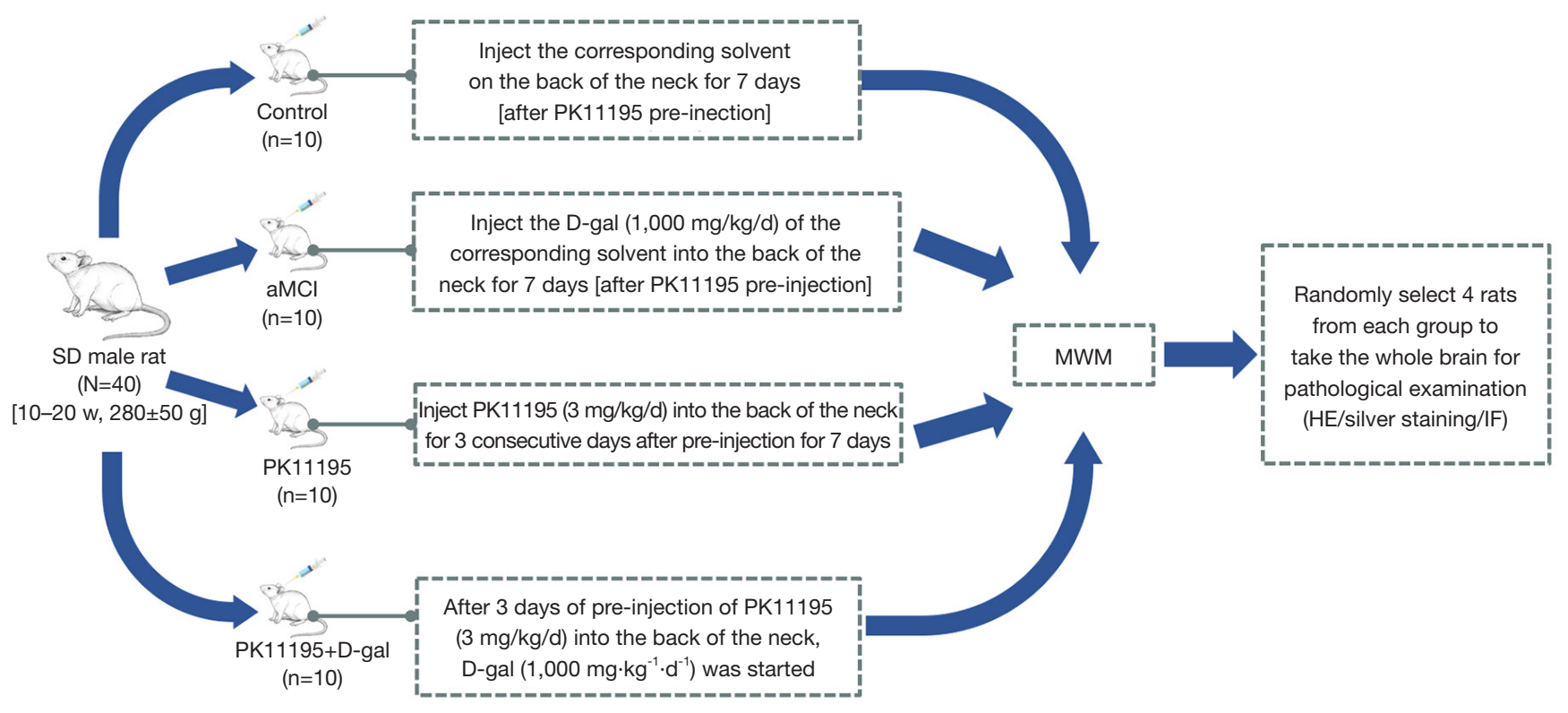

Figure 1 Experimental flow chart. aMCI, amnestic mild cognitive impairment; D-gal, D-galactose; MWM, Morris water maze; HE, hematoxylin-eosin staining; IF, immunofluorescence.

Experimental Animal Co., Ltd. [qualification certificate no.: SCXK (Shanghai) 2017-0005]. The animals were kept at the Experimental Animal Center of Fujian Medical University [SPF (specific-pathogen-free) level, license number: SYXK (Min) 2016-0006]. The rats were subjected to cognitive function behavioral tests in The Anesthesiology Laboratory of the First Affiliated Hospital of Fujian Medical University. The animals were given free access to food and water, under a 12-h light (6:00-18:00)/dark (18:00-6:00) cycle. The feeding room was kept at a constant temperature $\left(20-25^{\circ} \mathrm{C}\right)$ and humidity $(55 \%)$. The rats were given a 5 -day adaptation period before the experiment.

\section{Groups}

Rats were randomly assigned to the following groups according to body weight: the control group $(n=10)$, the D-gal group (aMCI group, $\mathrm{n}=10$ ), the PK11195 + D-gal group $(n=10)$, and the PK11195 group $(n=10)$. The PK11195 + D-gal and PK11195 groups were intraperitoneally injected with PK11195 (3 mg/kg)(11). Starting 3 days after the PKIII95 injection, the rats in the D-gal and PK11195 + D-gal groups were administered an injection of D-gal $\left(1,000 \mathrm{mg} \cdot \mathrm{kg}^{-1} \cdot \mathrm{D}^{-1}\right)$ via the head and neck for 7 consecutive days. The D-gal was dissolved in saline, and PK11195 was dissolved in saline containing $0.4 \%$
Tween 80 (flow chart, Figure 1).

\section{Behavioral testing}

The Morris water maze (MWM) was used to test for behavioral changes in the model rats. The MWM tests the learning and memory ability of rats based on their spatial positioning and sense of direction by forcing them to find a transparent platform hidden under the water surface. The 5 -day water maze navigation experiment was started 1 day after the end of building mode, and the space exploration experiment was conducted 1 day after the completion of navigation experiment.

\section{Patbology testing}

After the behavioral test, four rats from each group were selected at random. They were fasted for 12 hours but could drink water freely. The mice were then sacrificed, their brains were removed, and the brain tissues were immersed in the fixed solution for $24 \mathrm{~h}$ and then embedded in paraffin the next day. After confirming the anatomic location of hippocampus, serial sections were taken on coronal plane. Some sections were stained with hematoxylin eosin. The other part of the slices were immersed in silver solution, and then treated with reducing agent, the silver particles settled 
Table 1 Comparison of body weight (g) of the rats during the experiment $(\bar{x} \pm s, \mathrm{n}=10)$

\begin{tabular}{lcccc}
\hline Group & D1 & D3 & D5 & D7 \\
\hline Control & $305.43 \pm 8.33$ & $311.37 \pm 9.27$ & $317.49 \pm 5.40$ & $329.18 \pm 8.45$ \\
aMCl & $301.74 \pm 14.23$ & $308.76 \pm 16.50$ & $321.55 \pm 13.35$ & $326.90 \pm 16.01$ \\
PK11195 & $300.76 \pm 11.09$ & $314.82 \pm 11.62$ & $321.33 \pm 12.10$ & $330.57 \pm 13.06$ \\
PK11195 + D-gal & $302.56 \pm 15.07$ & $312.30 \pm 17.59$ & $324.29 \pm 16.66$ & $328.68 \pm 15.96$ \\
\hline
\end{tabular}

aMCl, amnestic mild cognitive impairment; D-gal, D-galactose.

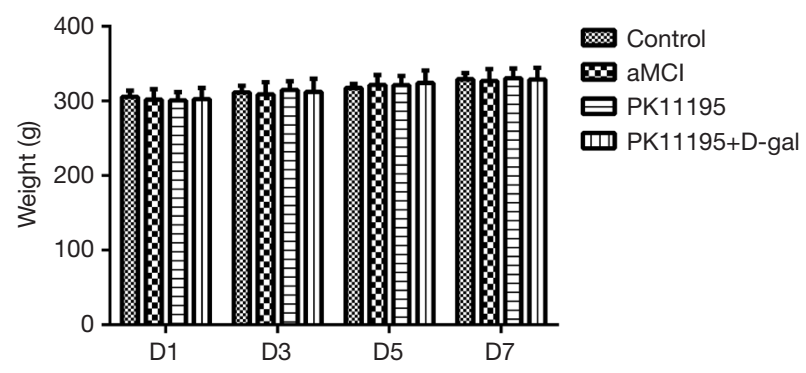

Figure 2 Weight comparison during the experiment $(\bar{x} \pm s, \mathrm{n}=10)$. aMCI, amnestic mild cognitive impairment; D-gal, D-galactose.

in the axonal plasm, making them black brown.

\section{Immunofluorescence (IF)}

Four rats were randomly selected from each group. They were fasted 12 hours but had free access to water. The mice were then sacrificed, and the brain tissue was collected and embedded in paraffin. Some paraffinized sections were randomly selected for dewaxing in water. The primary antibody was PBR antibody (Abcam, ab92291) with dilution ratio of 1:100; the secondary antibody was $\mathrm{Cy} 3$ antibody (Servicebio, gb21404) with dilution ratio of 1:200. After fluorescent staining, the slices were observed under a fluorescence microscope and the images were collected (the 4',6-diamidino-2-phenylindole (DAPI) ultraviolet (UV) excitation wavelength: $330-380 \mathrm{~nm}$, emission wavelength: $420 \mathrm{~nm}$, blue light; fluorescein isothiocyanate (FITC) excitation wavelength: $465-495 \mathrm{~nm}$, emission wavelength: 515-555 nm, green light; CY3 excitation wavelength: 510$560 \mathrm{~nm}$, emission wavelength: $590 \mathrm{~nm}$ ) .

\section{Statistical analysis}

Statistical analyses were performed with IBM SPSS STATISTICS 22.0(Armonk, NY: IBM Corp). The data are presented as mean \pm standard error. The data obtained from the MWM localization and navigation experiment during the escape latency period were analyzed using the repeated measures analysis of variance (ANOVA). One-way ANOVA was applied for the data from space exploration experiments and the results of immunofluorescence detection. The data of each group were first tested for normality and homogeneity of variance. Two-way ANOVA was used for the measurement data of body weight and swimming speed. The Bonferroni post-hoc test was used to explore differences between groups. $\mathrm{P}<0.05$ was considered statistically significant.

\section{Results}

\section{Animal characteristics}

\section{Weight changes in rats}

The rats in each group showed good activity and normal food and water consumption; none of the rats in these groups died. In each group, the weights of the rats in the early, middle, and late stages (i.e., days 1, 3, 5, and 7) of the drug intervention period conformed to a normal distribution. There were no statistically significant differences in the body weight of the rats on day $1,3,5$, or 7 (Table 1, Figure 2, $\mathrm{P}>0.05$ ).

\section{Changes in swimming speed}

The typical measurement results in the early, middle, and late stages (days 1,3 , and 5 , respectively) were selected for statistical analysis. The swimming speeds of the rats in each group on days 1,3 , and 5 were normally distributed. The results of the water maze experiment showed no statistically significant difference in the swimming speed of the rats in each group on day 1, 3, or 5 (Table 2, Figure 3, P>0.05).

\section{Place navigation test}

\section{Rat trajectory changes}

In the early stage of the MWM positioning experiment, 
Table 2 Comparison of swimming speed ( $\mathrm{mm} / \mathrm{s})$ between the groups of rats $(\bar{x} \pm s, \mathrm{n}=10)$

\begin{tabular}{lccc}
\hline Group & D1 & D3 & D5 \\
\hline Control & $205.95 \pm 17.05$ & $195.15 \pm 20.07$ & $201.14 \pm 28.42$ \\
aMCl & $217.19 \pm 12.78$ & $185.26 \pm 13.03$ & $198.00 \pm 29.46$ \\
PK11195 & $205.22 \pm 6.98$ & $200.77 \pm 16.78$ & $179.18 \pm 15.03$ \\
PK11195 + D-gal & $216.08 \pm 15.90$ & $204.75 \pm 35.39$ & $203.07 \pm 32.60$ \\
\hline
\end{tabular}

aMCl, amnestic mild cognitive impairment; D-gal, D-galactose.

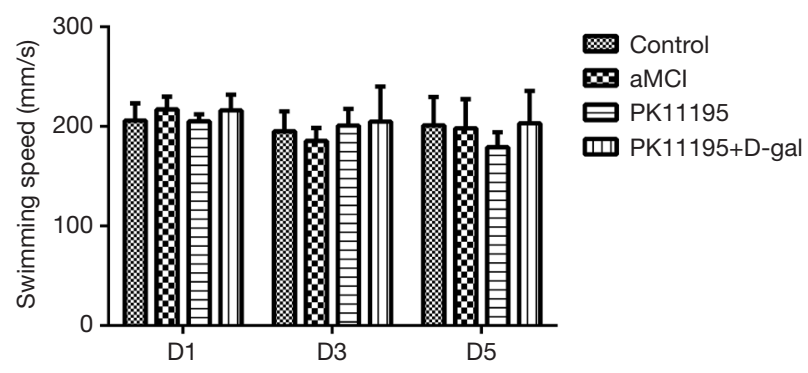

Figure 3 Swimming speed $(\mathrm{mm} / \mathrm{s})$ comparison $(\bar{x} \pm s, \mathrm{n}=10)$. aMCI, amnestic mild cognitive impairment; D-gal, D-galactose.

compared with the control group, the aMCI group's behavior of circumventing the wall was more obvious, which was mainly represented by the extension of the movement path and the reduction of the number of crossings in the middle area of the quadrant. In the early stage, the model rats pre-treated with PK11195 occasionally traversed the middle area of the quadrant. In the middle and late stages, the PK11195 group could quickly find the platform, and the behavior path was similar to that of the control group, while the PK11195 + D-gal group's motion path was longer than that of the control group. Meanwhile, compared to the aMCI group, the PK11195 group took a shorter time to find the platform and crossed the middle area of the quadrant more times, with a straighter and single trajectory. During the experiment, the main movement modes were: "edge type $\rightarrow$ random type $\rightarrow$ trend type" in the aMCI group; "trend type $\rightarrow$ linear type" or "random type $\rightarrow$ trend type $\rightarrow$ linear type" in the control group and the PK11196 group; and "edge type $\rightarrow$ random type" or "random type $\rightarrow$ trending type $\rightarrow$ linear type" in the PK11195 + D-gal group (Figure 4).

\section{Changes in rat escape latency}

The results of the escape latency test differed between the groups (Table 3, Figure 5, $\mathrm{F}=11.661, \mathrm{P}<0.001$ ).
Multiple comparisons [least significant difference (LSD) $t$-tests] showed that there were no statistically significant differences between the control group and the PK11195 + $\mathrm{D}$-gal group $(\mathrm{P}>0.05)$. Compared with the control group, the aMCI group had prolonged escape latency $(\mathrm{t}=-5.337$, $\left.{ }^{*} \mathrm{P}<0.05\right)$. Moreover, the escape latency of the PK11195 group was shorter than that of the control group ( $t=2.255$, $\left.{ }^{*} \mathrm{P}<0.05\right)$.

\section{Spatial probe test}

The number of platform crossings was lower in the aMCI group than in the control group $\left({ }^{*} \mathrm{P}<0.05\right)$; however, there was no significant difference with the number of platform crossings in the PK11195 group ( $\mathrm{P}>0.05)$. The PK11195 $+\mathrm{D}$-gal group had a higher number of platform crossings than the aMCI group $\left({ }^{*} \mathrm{P}=0.019,{ }^{\#} \mathrm{P}<0.05\right)$ (Table 4, Figure 6).

\section{Pathological changes in hippocampal CA1 area of rat brain}

\section{HE staining}

\section{Histological appearance of pyramidal cells at low magnification}

Figure 7 shows a schematic diagram of the arrangement of pyramidal cells in the HE stained sections of the hippocampal CA1 area of rats in the control group, aMCI group, PK11195 group, and PK11195 + D-gal group. Among them, the Figure $7 A, B, C, D$ show the $10-\times 10$-fold microscopic observations of the above experimental groups, respectively. As shown in the diagram, the number and size of the pyramidal cells in the hippocampal CA1 region of rats in the control group and PK11195 group were found to be normal, with uniform and orderly arrangement, and normal density. However, under the microscope, the number of pyramidal cells in the CA1 region of rats in the aMCI group was reduced, and some of the pyramidal cells differed in size. The overall arrangement of pyramidal cells was relatively sparse and disorderly, with a reduced density. 


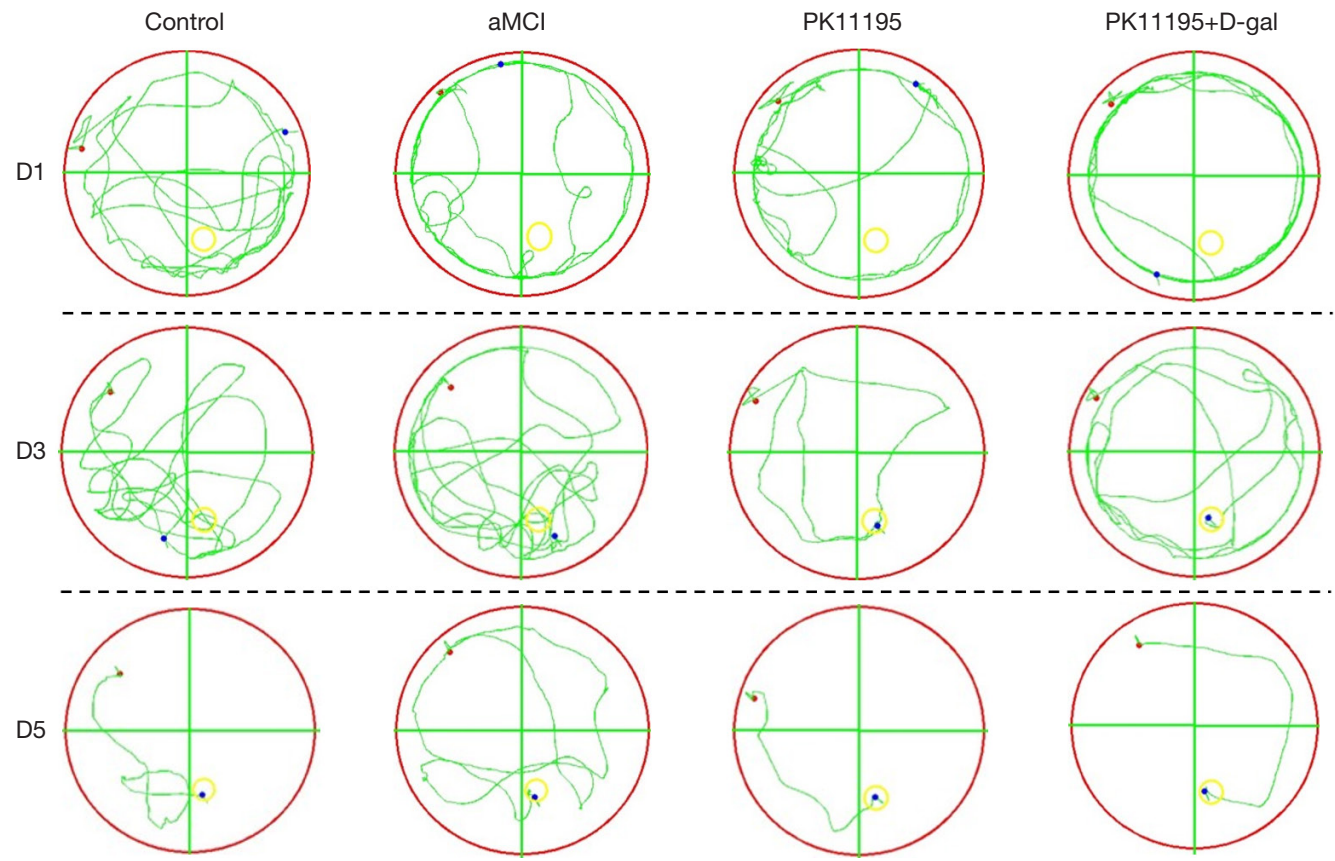

Figure 4 Trajectories of the groups in the water maze navigation experiment. aMCI, amnestic mild cognitive impairment; D-gal, D-galactose.

Table 3 Comparison of escape latency between the groups of rats $(\bar{x} \pm s, \mathrm{n}=10)$

\begin{tabular}{lccccc}
\hline Group & D1 & D2 & D3 & D4 & D5 \\
\hline Control & $59.38 \pm 8.18$ & $48.35 \pm 10.29$ & $34.51 \pm 9.90$ & $21.57 \pm 4.95$ & $11.39 \pm 2.11$ \\
aMCl & $79.06 \pm 8.32^{\star}$ & $63.61 \pm 11.26^{\star}$ & $32.01 \pm 7.31^{\star}$ & $18.99 \pm 5.39^{\star}$ & $17.90 \pm 5.35^{\star}$ \\
PK11195 & $58.04 \pm 7.76^{\star}$ & $35.55 \pm 7.21^{\star}$ & $23.68 \pm 13.85^{\star}$ & $16.75 \pm 7.57^{\star}$ & $9.08 \pm 2.46^{\star}$ \\
PK11195 + D-gal & $59.31 \pm 11.23$ & $50.82 \pm 11.23$ & $35.64 \pm 11.46$ & $14.94 \pm 5.41$ & $8.95 \pm 2.32$ \\
\hline
\end{tabular}

*, compared with control group, $\mathrm{P}<0.05$. aMCl, amnestic mild cognitive impairment; D-gal, D-galactose.

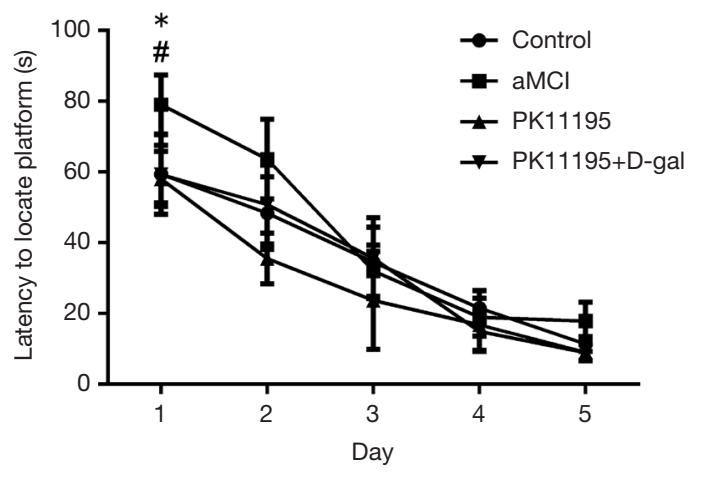

Figure 5 Comparison of escape latency in the groups of rats $(\bar{x} \pm s$, $\mathrm{n}=10) .{ }^{*}$, compared with the control group, $\mathrm{P}<0.05$; *, compared with the aMCI group, $\mathrm{P}<0.05$. aMCI, amnestic mild cognitive impairment; D-gal, D-galactose.
Table 4 The number of platform crossings by each group of rats $(\bar{x} \pm s, \mathrm{n}=10)$

\begin{tabular}{lc}
\hline Group & Number of platform crossings \\
\hline Control & $3.60 \pm 1.95$ \\
aMCl & $1.60 \pm 0.97^{*}$ \\
PK11195 & $3.60 \pm 2.17^{\#}$ \\
PK11195 + D-gal & $3.50 \pm 1.96^{\#}$ \\
\hline${ }^{*}$, compared with control group, P<0.05; ${ }^{\#}$, compared with aMCl \\
group, P<0.05. aMCl, amnestic mild cognitive impairment; D-gal, \\
D-galactose.
\end{tabular}


In the PK11195 + D-gal group, the number of pyramidal cells in the CA1 region was normal, as was the overall density, and some of the cells had an abnormal size and disordered arrangement.

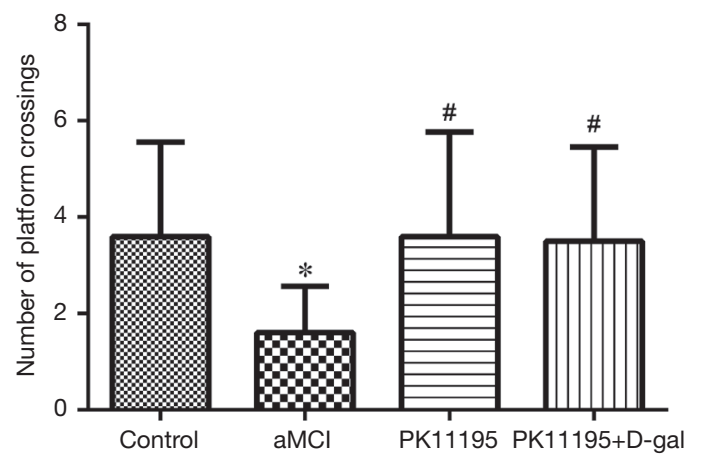

Figure 6 The number of platform crossings by each group of rats $(\bar{x} \pm s, \mathrm{n}=10) .{ }^{*}$, compared with the control group, $\mathrm{P}<0.05$; \#, compared with the aMCI group, $\mathrm{P}<0.05$. aMCI, amnestic mild cognitive impairment; D-gal, D-galactose.

\section{Histological appearance of pyramidal cells at high magnification}

At a magnification of $40 \times 10$ (Figure 8), the structure of pyramidal cells in the control and the PK11195 groups was complete, the cytoplasm was uniform, the nuclei were centered, and the nucleoli were obvious. In contrast, some of the pyramidal cells in the CA1 region of rats in the aMCI group were structurally damaged, the cytoplasm was heterogeneous and even vacuolated, and the nuclei of the cells were hyperchromatic, and some of the cells showed a state of apoptosis. Although some of the pyramidal cells in the PK11195 pretreatment group showed structural damage, the rest of the cells maintained normal cell structure, the cytoplasm was uniform, the nucleoli were obvious, and vacuolization was rare.

\section{Silver staining}

Histological appearance of astrocytes at low magnification Figure 9 shows a schematic diagram of the arrangement of astrocytes in the gray matter near the CA1 region of the hippocampal tissues of the control group, aMCI group,
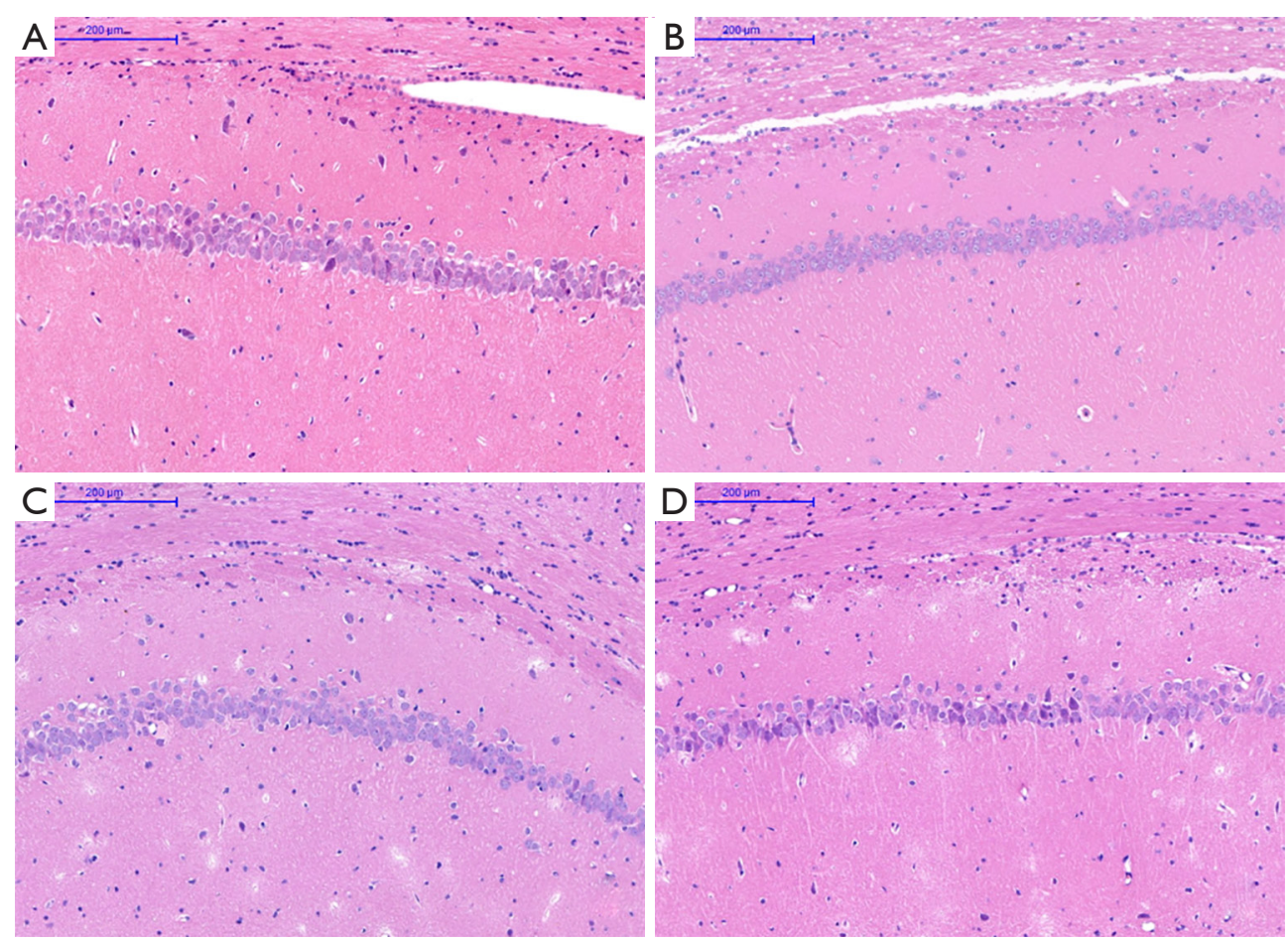

Figure 7 Pathological changes in pyramidal cells in the CA1 region $(10 \times 10)$. (A,B,C,D) represent the pathological changes of the pyramidal cells (blue-purple) in the hippocampal CA1 region of the rats from different experimental groups under $10 \times 10$ magnification. (A) is the control group, (B) is the aMCI group, (C) is the PK11195 group, and (D) is the PK11195 + D-gal group. aMCI, amnestic mild cognitive impairment; D-gal, D-galactose. 

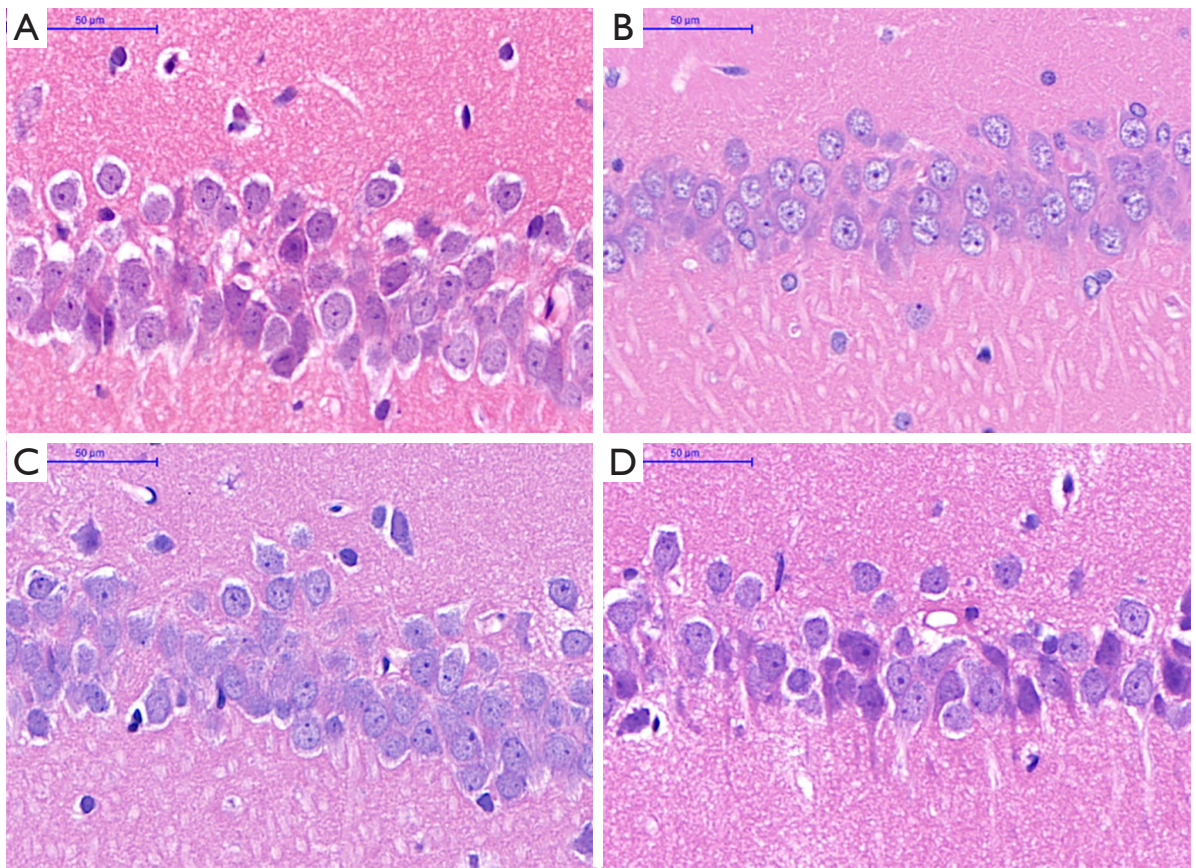

Figure 8 Morphological changes of pyramidal cells in CA1 region $(40 \times 10)$. (A,B,C,D) represent the pathological changes of the pyramidal cells (blue-purple) and intracellular structure of the hippocampal CA1 region of the different experimental groups under $40 \times 10$ magnification. (A) is the control group, (B) is the aMCI group, (C) is the PK11195 group, and (D) is the PK11195 + D-gal group. aMCI, amnestic mild cognitive impairment; D-gal, D-galactose.
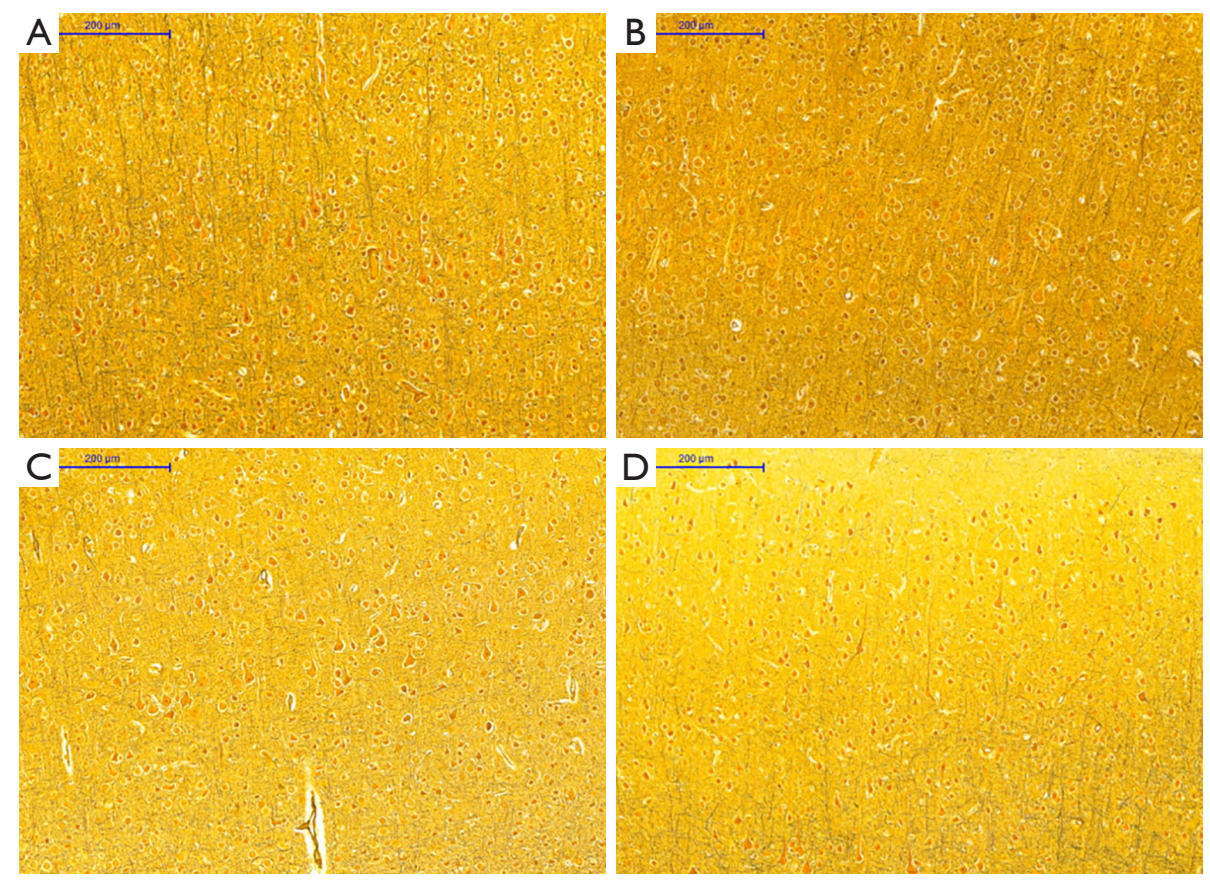

Figure 9 Pathological changes of astrocytes in gray matter near CA1 region $(10 \times 10)$. (A,B,C,D) represent the pathological changes of astrocytes (black-brown) in the gray matter of the hippocampus in the different experimental groups under $10 \times 10$ magnification. (A) is the control group, (B) is the aMCI group, (C) is the PK11195 group, and (D) is the PK11195 + D-gal group. aMCI, amnestic mild cognitive impairment; D-gal, D-galactose. 

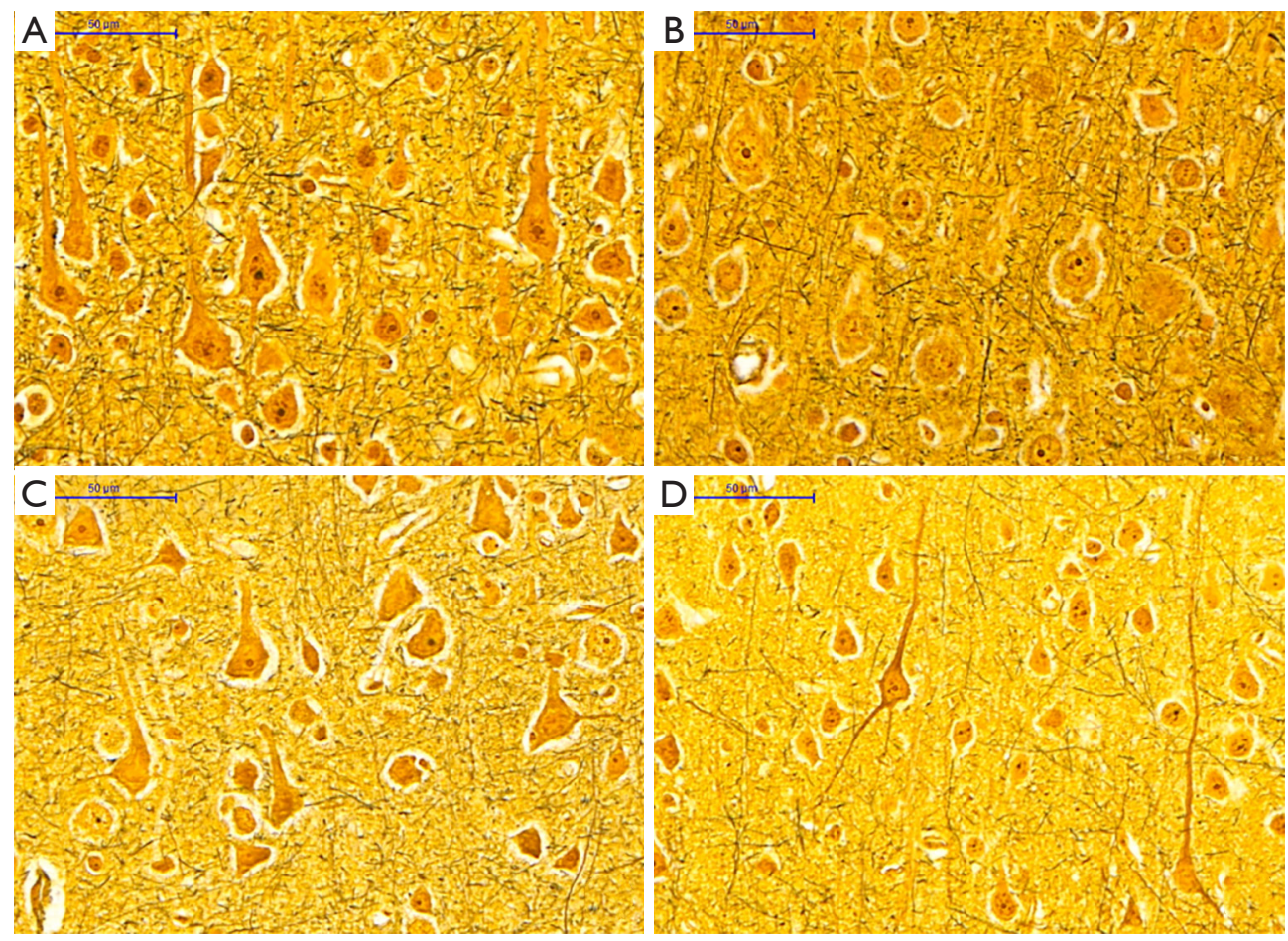

Figure 10 Pathological changes of astrocytes in gray matter near CA1 region $(40 \times 10)$. (A,B,C,D) represent the pathological changes of astrocytes (black-brown) in the gray matter near the hippocampal CA1 region of the different experimental groups under $40 \times 10$ magnification. (A) is the control group, (B) is the aMCI group, (C) is the PK11195 group, and (D) is the PK11195 + D-gal group. aMCI, amnestic mild cognitive impairment; D-gal, D-galactose.

PK11195 group, and PK11195 + D-gal group under 10×10 magnification (Figure 9). The astrocytes in the control and PK11195 groups were normal in number and density, neatly arranged, and evenly distributed. In the aMCI group, the astrocytes were decreased in number and density, and unevenly distributed. In the PK11195 + D-gal group, the number of astrocytes in the gray matter near the CA1 region was not significantly reduced, the density was slightly reduced, and the arrangement was neater compared to those in the aMCI group.

\section{Histological appearance of astrocytes at high magnification}

In the control and PK11195 groups, the gray matter in the brain tissue was near the CA1 region, and the astrocyte nuclei were mostly round or oval in shape, with dark staining. The glial filaments in the cytoplasm were interwoven, and many protrusions ran radially on the outside of the cell, with an enlarged foot plate seen at the end of some protrusions. However, in the aMCI group, the cytoplasm of the astrocytes was lightly stained, some glial cell nucleoli had disappeared, and the cytoplasmic structure was not clear; also, the glial filaments were reduced and the protrusions around the cell body were missing. In the PK11195 + D-gal group, the morphology and structure of the astrocytes were relatively normal with deep staining. The density of glial filaments in the cytoplasm was normal. Some cell protrusions were missing; however, the cell structure was still clear (Figure 10).

\section{Distribution of TSPO expression by immunofluorescence}

The expression and location of TSPO in the hippocampus of the aMCI group rats, along with the effect of PK11195 on TSPO protein expression, were further investigated by immunofluorescence (Figures 11,12). In the aMCI group, a large amount of TSPO protein was expressed in the pyramidal cell membrane in the hippocampal CA1 region of the brain, which showed bright red fluorescence. At least three 200 -fold fields of vision were randomly selected for each slice in each group for semi-quantitative analysis (Figure 13). Compared with the control group, the expression of TSPO in the hippocampal CA1 region of the aMCI group was significantly higher than that in $(\mathrm{F}=30.547$, 

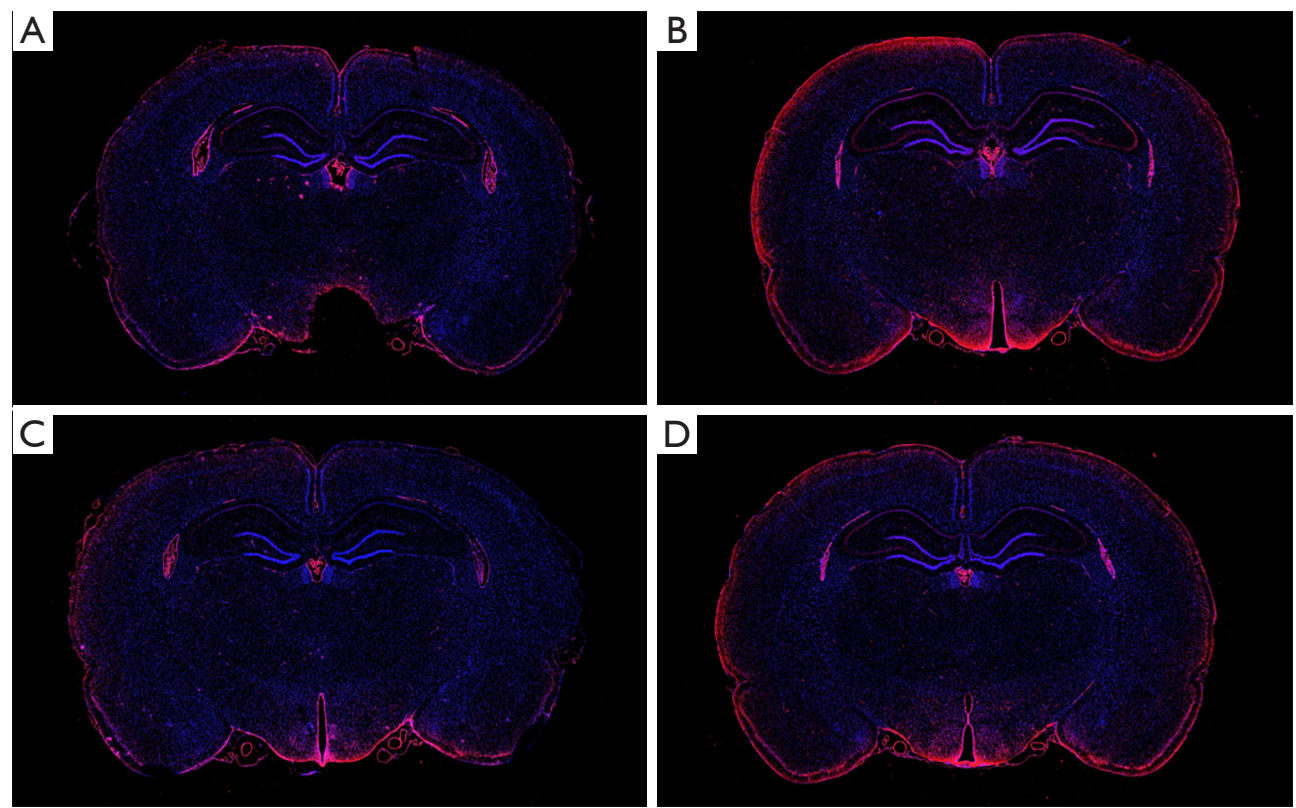

Figure 11 Coronal scans of slices of rat brain stained by immunofluorescence. (A,B,C,D) represent the control group, aMCI group, PK11195 group, and PK11195 + D-gal group respectively. The nuclei were stained with DAPI (4',6-diamidino-2-phenylindole) blue and TSPO with red staining. The TSPO expression area of brain tissue in the aMCI group was wide and the fluorescence density was high; the expression range of TSPO in aMCI rats after PK11195 pretreatment became narrower and the fluorescence density decreased. aMCI, amnestic mild cognitive impairment; D-gal, D-galactose.

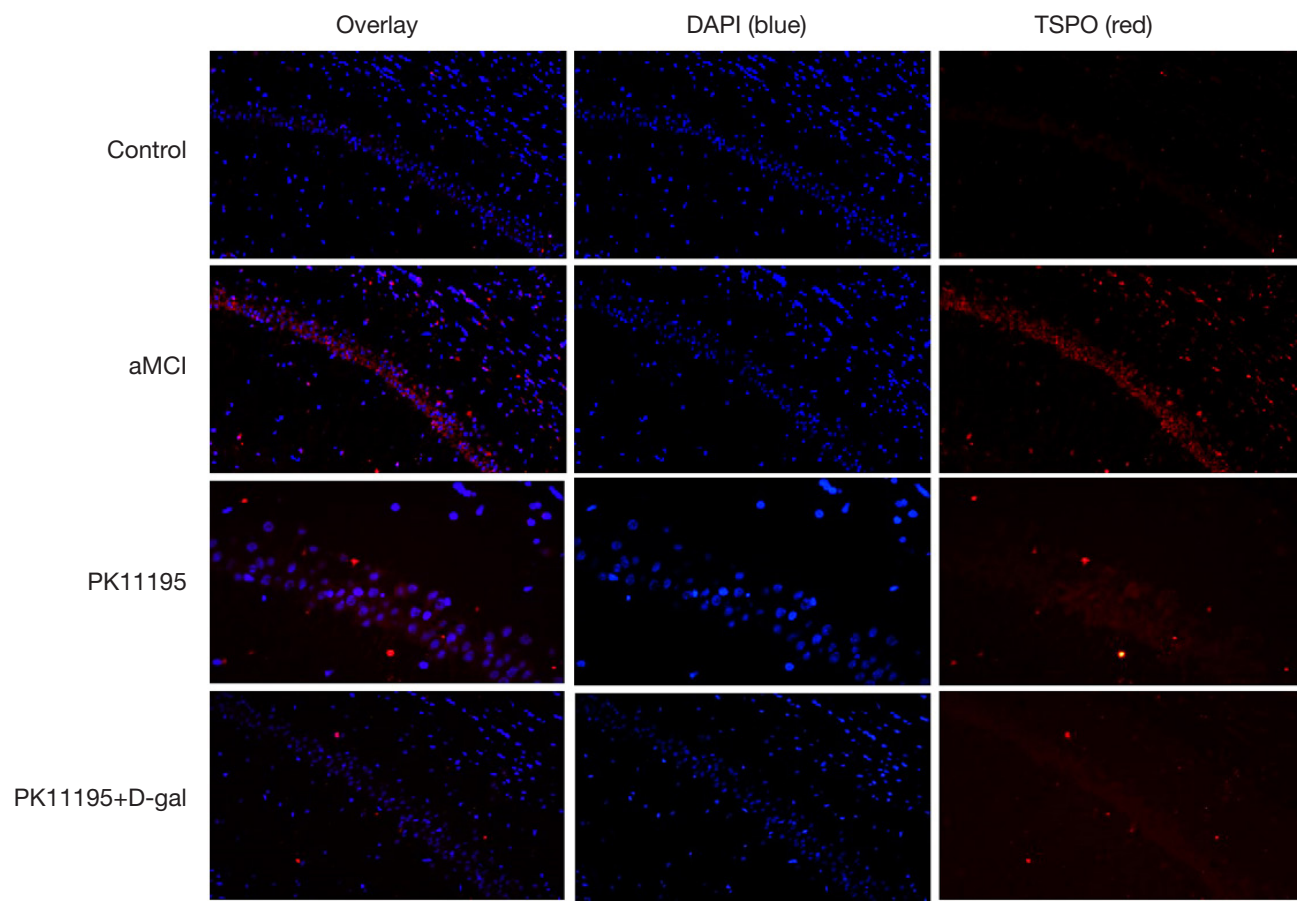

Figure 12 Immunofluorescence scan of the hippocampal CA1 region $(40 \times 10)$. The effect of PK11195 on TSPO expression and location in the hippocampal CA1 region. The nuclei were stained with DAPI (4',6-diamidino-2-phenylindole)blue and TSPO with red staining. aMCI, amnestic mild cognitive impairment; D-gal, D-galactose. 


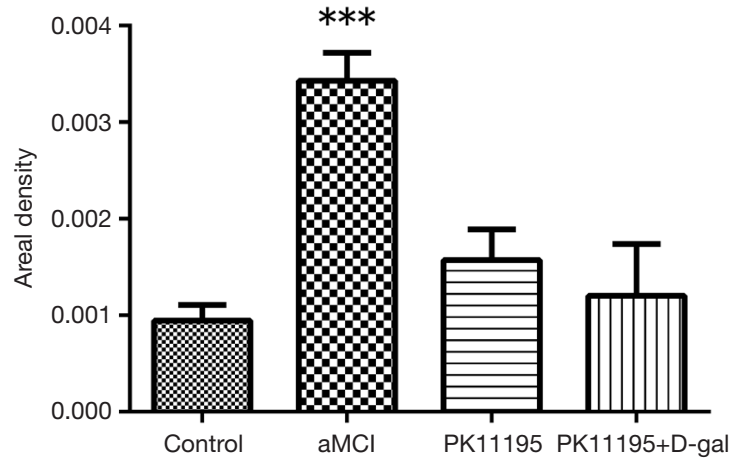

Figure 13 Analysis of TSPO expression in the hippocampal CA1 region $(\bar{x} \pm s, \mathrm{n}=4)$. ${ }^{* *}$, compared with the control group, $\mathrm{P}<0.001$. aMCI, amnestic mild cognitive impairment; D-gal, D-galactose.

$\left.{ }^{* * *} \mathrm{P}<0.001\right)$. However, the expression of TSPO protein around the pyramidal cell membrane in the hippocampal CA1 area of the PK11195 + D-gal group was not statistically different from that in the control group $(\mathrm{P}>0.05)$, and the expression of TSPO protein in the PK11195 group was not statistically different from that in the control group $(\mathrm{P}>0.05)$.

\section{Discussion}

TSPO-related physiological functions have mainly been studied through TSPO ligands, which are closely related to the occurrence and development of central neurodegenerative diseases. TSPO ligands are mainly divided into endogenous and exogenous ligands, among which cholesterol and porphyrins are significant. $A \beta$ protein is known to play a major pathological role in the brains of $\mathrm{AD}$ patients; astrocytes can induce endogenous TSPO ligands after induction by $\mathrm{A} \beta$ protein (21), which means endogenous TSPO ligands can be used as biomarkers. Various neuropathological studies have been conducted on brain aging and reactive glial hyperplasia. In addition to the pathological diagnosis of endogenous ligands, recent studies have shown that exogenous TSPO ligands can be used for neuroprotection, as well as to limit neuroinflammation, promote glial cell regeneration, and treat nervous system dysfunction (22). Among these exogenous ligands, PK11195 and Ro5-4864 have been shown to exert multiple neuroprotective effects and have been commonly used in the field of brain-related cognitive function research (23). PK11195 can specifically bind to TSPO, whereas Ro5-4864 requires the assistance of other mitochondrial proteins (24). In view of this difference in binding function between the two ligands, this experiment used PK11195 to conduct a more direct and concise mechanism study.

Previous studies confirmed that mitochondrial damage is an important initiating factor leading to cell apoptosis (25). The dysfunction of mitochondrial damage in relevant nerve cells in the brain leads to nerve cell damage in the learning and memory brain region, and eventually leads to cognitive dysfunction. When D-gal and other pro aging factors lead to astrocyte damage, the increase of TSPO protein expression in the local area will lead to the disorder of mitochondrial cholesterol synthesis, which will affect the function of mitochondrial synthesis and metabolism, and then lead to a large number of apoptotic substances such as AIF, Cyt C, and release to the cytoplasm, and finally accelerate the apoptosis of astrocyte. Previous studies have shown that excessive activation of glial cells (mainly microglia) in the process of brain injury leads to a large number of TSPO expression (26), and ultimately accelerates cognitive impairment. With the accumulation of TSPO, mitochondria in astrocyte release apoptosis related inducing factors, which changes the structure and biological function of astrocyte. After TSPO is activated by aging factors, the transport of TSPO dependent cholesterol to mitochondria is accelerated, which results in the impairment of cholesterol synthesis function and further leads to neurological dysfunction

The immunofluorescence results of this study showed that the expression areas of TSPO in the hippocampal CA1 region of rat hippocampus tissue after PK11195 pretreatment were narrower than that in aMCI group. During the experiment, the body weight of the rats did not fluctuate significantly during intervention with PK11195, nor did the swimming speed of the rats change abnormally, which indicates that PK11195 did not affect the normal limb motor function of the rats. During the behavior test, the ability of the rats in the control, PK11195, and PK11195 + $\mathrm{D}$-gal groups to find the platform increased continuously with training time, and the time required to locate the platform became shorter over time, indicating that PK11195 effectively relieved D-gal-induced learning and memory dysfunction. Compared with the rats in the aMCI group without intervention, the number of times the rats in the PK11195 + D-gal group traversed the platform increased, indicating that their spatial recognition and memory function had recovered; meanwhile, compared with the normal rats, the number of platform crossings in the PK11195 group did not increase, suggesting that injection of PK11195 can alleviate the mild learning and memory function decline 
caused by D-gal without affecting the normal memory and learning ability of the brain. The pathological results showed that PK11195 can prevent the pathological changes of pyramidal cells caused by D-gal, maintain the normal morphological structure of pyramidal cells, and delay the processes of cell damage and apoptosis caused by D-gal. Immunoblotting experiments further revealed that the expression of TSPO in the hippocampus of rats in the PK11195 + D-gal group was lower than that in the aMCI group. The expression of TSPO is generally believed to significantly increase with AS damage or microglia activation in brain tissue and can be used as a sensitive indicator to judge the functional integrity of mitochondria in the brain. Compared with the rats in the aMCI group, the expression of TSPO in the hippocampus of rats in the PK11195 + D-gal group was significantly decreased, confirming that PK11195 can significantly inhibit the D-gal-induced expression of TSPO protein in the hippocampus. Furthermore, compared with the normal rats, no significant difference was observed in TSPO expression in the hippocampus of the PK11195 group rats, suggesting that PK11195 alone did not affect the normal physiological expression of TSPO.

\section{Conclusions}

The findings of the present study can be summarized as follows: (I) the exogenous TSPO ligand PK11195 can alleviate D-gal-induced cognitive impairment associated with early learning and memory function; (II) PK11195 can prevent the pathological changes of astrocytes and pyramidal cells by reducing the high expression of TSPO induced by $\mathrm{D}$-gal in the hippocampus, and alleviate early decline in learning and memory function. It has been shown that voltage dependent anion channel (VDAC) and mitochondria inner membrane protein adenine nucleotide translocator (ANT) constitute the core of mitochondrial multiprotein complex (MPTP) (27). Since TSPO is located in the outer membrane of mitochondria and is associated with VDAC, it suggests that TSPO may be related to MPTP; at the same time, the loss of mitochondrial endomembrane potential in response to apoptosis induction can be prevented by directly regulating TSPO to regulate MPTP complex (28). The mechanism of PK11195 in preventing as induced by $\mathrm{D}$-gal may involve the change of binding function between TSPO and MPTP.

In this experiment, the expression of TSPO in the hippocampus of the aMCI group was found to be abnormally increased. At the same time, the results of immunofluorescence showed that D-gal induced early decline in learning and memory function along with abnormal expression of TSPO in the hippocampus, and other brain areas also showed increased expression of TSPO. Our results suggest that expression of TSPO in the brain tissue of amnestic cognitive impairment is increased, which can lead to cognitive behavior abnormalities

\section{Acknowledgments}

Funding: This work was supported by the Natural Science Foundation of Fujian Province, China (Grant No. 2018J01178) and the Project of Fujian Medical Innovation (Grant No. 2015-CX-22).

\section{Footnote}

Reporting Checklist: The authors have completed the ARRIVE reporting checklist. Available at http://dx.doi. org/10.21037/atm-20-6157

Data Sharing Statement: Available at http://dx.doi. org/10.21037/atm-20-6157

Conflicts of Interest: All authors have completed the ICMJE uniform disclosure form (available at http://dx.doi. org/10.21037/atm-20-6157). The authors have no conflicts of interest to declare.

Etbical Statement: The authors are accountable for all aspects of the work in ensuring that questions related to the accuracy or integrity of any part of the work are appropriately investigated and resolved. Experiments were performed under a project license (No.: 2018-321) granted by Fujian experimental animal association, in compliance with EU Directive 2010/63/EU for the care and use of animals,

Open Access Statement: This is an Open Access article distributed in accordance with the Creative Commons Attribution-NonCommercial-NoDerivs 4.0 International License (CC BY-NC-ND 4.0), which permits the noncommercial replication and distribution of the article with the strict proviso that no changes or edits are made and the original work is properly cited (including links to both the formal publication through the relevant DOI and the license). See: https://creativecommons.org/licenses/by-nc-nd/4.0/. 


\section{References}

1. Albert MS, DeKosky ST, Dickson D, et al. The diagnosis of mild cognitive impairment due to Alzheimer's disease: recommendations from the National Institute on AgingAlzheimer's Association workgroups on diagnostic guidelines for Alzheimer's disease. Alzheimers Dement 2011;7:270-9.

2. Ilhan Algin D, Dagli Atalay S, Ozkan S, et al. Memantine improves semantic memory in patients with amnestic mild cognitive impairment: A single-photon emission computed tomography study. J Int Med Res 2017;45:2053-64.

3. Huang YP, Singh A, Chen S, et al. Validity of a Novel Touch Screen Tablet-Based Assessment for Mild Cognitive Impairment and Probable AD in Older Adults. Assessment 2019;26:1540-53.

4. Assoc, A. s.2018 Alzheimer's disease facts and figures. Alzheimers \& Dementia 2018;14:367.

5. Xian YF, Lin ZX, Zhao M, et al. Uncaria rhynchophylla ameliorates cognitive deficits induced by D-galactose in mice. Planta Med 2011;77:1977-83.

6. Zhu J, Mu X, Zeng J, et al. Ginsenoside Rg1 prevents cognitive impairment and hippocampus senescence in a rat model of D-galactose-induced aging. PLoS One 2014;9:e101291.

7. Li H, Sagar AP, Kéri S. Translocator protein $(18 \mathrm{kDa}$ TSPO) binding, a marker of microglia, is reduced in major depression during cognitive-behavioral therapy. Prog Neuropsychopharmacol Biol Psychiatry 2018;83:1-7.

8. Hauet T, Han Z, Wang Y, et al. Modulation of peripheral-type benzodiazepine receptor levels in a reperfusion injury pig kidney-graft model. Transplantation 2002;74:1507-15.

9. Papadopoulos V, Baraldi M, Guilarte TR, et al. Translocator protein $(18 \mathrm{kDa})$ : new nomenclature for the peripheral-type benzodiazepine receptor based on its structure and molecular function. Trends Pharmacol Sci 2006;27:402-9.

10. Nicastro N, Surendranathan A, Mak E, et al. 11 C-PK11195 PET imaging and white matter changes in Parkinson's disease dementia. Ann Clin Transl Neurol 2019;6:2133-6.

11. Barron AM, Garcia-Segura LM, Caruso D, et al. Ligand for translocator protein reverses pathology in a mouse model of Alzheimer's disease. J Neurosci 2013;33:8891-7.

12. Veiga S, Carrero P, Pernia O, et al. Translocator protein
$18 \mathrm{kDa}$ is involved in the regulation of reactive gliosis. Glia. 2007;55:1426-36.

13. Ma L, Zhang H, Liu N, et al. TSPO ligand PK11195 alleviates neuroinflammation and beta-amyloid generation induced by systemic LPS administration. Brain Res Bull 2016;121:192-200.

14. Fukudome D, Hayes LN, Faust TE, et al. Translocator protein (TSPO) and stress cascades in mouse models of psychosis with inflammatory disturbances. Schizophr Res 2018;197:492-7.

15. Distler C, Dreher Z, Stone J. Contact spacing among astrocytes in the central nervous system: an hypothesis of their structural role. Glia 1991;4:484-94.

16. Li J, Khankan RR, Caneda C, et al. Astrocyte-toastrocyte contact and a positive feedback loop of growth factor signaling regulate astrocyte maturation. Glia 2019;67:1571-97.

17. Galli E, Rossi J, Neumann T, et al. Mesencephalic Astrocyte-Derived Neurotrophic Factor Is Upregulated with Therapeutic Fasting in Humans and Diet Fat Withdrawal in Obese Mice. Sci Rep 2019;9:14318.

18. Steele ML, Fuller S, Maczurek AE, et al. Chronic inflammation alters production and release of glutathione and related thiols in human U373 astroglial cells. Cell Mol Neurobiol 2013;33:19-30.

19. Niedzwiecka K, Tisi R, Penna S, et al. Two mutations in mitochondrial ATP6 gene of ATP synthase, related to human cancer, affect ROS, calcium homeostasis and mitochondrial permeability transition in yeast. Biochim Biophys Acta Mol Cell Res 2018;1865:117-31.

20. Madungwe NB, Feng Y, Lie M, et al. Mitochondrial inner membrane protein (mitofilin) knockdown induces cell death by apoptosis via an AIF-PARP-dependent mechanism and cell cycle arrest. Am J Physiol Cell Physiol 2018;315:C28-C43.

21. Zürcher NR, Loggia ML, Lawson R, et al. Increased in vivo glial activation in patients with amyotrophic lateral sclerosis: assessed with [(11)C]-PBR28. Neuroimage Clin 2015;7:409-14.

22. Bader S, Wolf L, Milenkovic VM, et al. Differential effects of TSPO ligands on mitochondrial function in mouse microglia cells. Psychoneuroendocrinology 2019;106:65-76.

23. Haider S, Liaquat L, Shahzad S, et al. A high dose of short term exogenous D-galactose administration in young male rats produces symptoms simulating the natural aging process. Life Sci 2015;124:110-9.

24. Ma B, Liu X, Huang X, et al. Translocator protein agonist Ro5-4864 alleviates neuropathic pain and 
Page 14 of 14

promotes remyelination in the sciatic nerve. Mol Pain 2018;14:1744806917748019.

25. Xu B, Lang LM, Li SZ, et al. Cortisol excess-mediated mitochondrial damage induced hippocampal neuronal apoptosis in mice following cold exposure. Cells 2019;8:612.

26. Mages K, Grassmann F, Jägle H, et al. The agonistic TSPO ligand XBD173 attenuates the glial response thereby protecting inner retinal neurons in a murine model
Ye et al. PK11195 and amnestic mild cognitive impairment

of retinal ischemia. J Neuroinflammation 2019;16:43.

27. Casellas P, Galiegue S, Basile AS. Peripheral benzodiazepine receptors and mitochondrial function. Neurochem Int 2002;40:475-86.

28. Everett H, Barry M, Sun X, et al. The myxoma poxvirus protein, M11L, prevents apoptosis by direct interaction with the mitochondrial permeability transition pore. J Exp Med 2002;196:1127-39.
Cite this article as: Ye C, Lin L, Zhang P, Chen Y, Huang J, Lin X. The protective effect of PK11195 on D-galactose-induced amnestic mild cognitive impairment in rats. Ann Transl Med 2020;8(18):1190. doi: 10.21037/atm-206157 